\title{
Popliteal Artery Above the Knee
}

National Cancer Institute

\section{Source}

National Cancer Institute. Popliteal Artery Above the Knee. NCI Thesaurus. Code

C116180.

The segment of the popliteal artery that is located above the knee. 